\title{
Wavelength Dependence of Light-Induced Cycloadditions
}

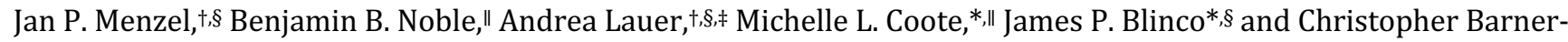 \\ Kowollik*,t,‡,§
}

§School of Chemistry, Physics and Mechanical Engineering, Queensland University of Technology (QUT), Brisbane, QLD 4000, Australia

†Macromolecular Architectures, Institut für Technische Chemie und Polymerchemie, Karlsruhe Institute of Technology (KIT), Engesserstr. 18, 76131 Karlsruhe, Germany

‡Institut für Biologische Grenzflächen, Soft Matter Synthesis Laboratory, Karlsruhe Institute of Technology (KIT), Hermann-von-Helmholtz-Platz 1, 76344 Eggenstein-Leopoldshafen, Germany

"Australian Research Council Centre of Excellence for Electromaterials Science, Research School of Chemistry, Australian National University, Canberra, ACT 2601, Australia

Wavelength dependent photochemistry, theoretical photochemistry, photoinduced cycloadditions, monochromatic tunable laser, action plot, relative quantitative mass spectrometry, numeric computational mass spectra evaluation.

ABSTRACT: The wavelength-dependent conversion of two rapid photoinduced ligation reactions, i.e. the light activation of $o$-methylbenzaldehydes, leading to the formation of reactive $o$-quinodimethanes (photoenols) and the photolysis of 2,5-diphenyltetrazoles, affording highly reactive nitrile imines, is probed via a monochromatic wavelength scan at constant photon count. The transient species are trapped by cycloaddition with $\mathrm{N}$-ethylmaleimide and conversion is traced by high resolution mass spectrometry and nuclear magnetic resonance spectroscopy. The resulting conversion vs. wavelength correlations are assessed in the context of Beer-Lambert's law and provide combined with time-dependent density functional theory and multireference calculations an in-depth understanding of the underpinning mechanistic processes, including conical intersections. The $\pi \rightarrow \pi^{*}$ transition of the carbonyl group of $o$ methylbenzaldehyde correlates with a highly efficient conversion to the cycloadduct, showing no significant wavelength dependence, while conversion following the $\mathrm{n} \rightarrow \pi^{*}$ transition proceed markedly less efficient at longer wavelengths. The influence of absorbance and reactivity has critical consequences for an effective reaction design: At high concentrations of $o$-methylbenzaldehydes $\left(c=8 \mathrm{mmol} \mathrm{L}^{-1}\right)$, photoligations with N-ethylmaleimide (possible for $\lambda \leq$ $390 \mathrm{~nm})$ are ideally performed at $330 \mathrm{~nm}$, whereas at high light penetration regimes at lower concentrations $(c=0.3$ mmol L-1), $315 \mathrm{~nm}$ irradiation leads to the highest conversion. Activation and trapping of 2,5-diphenyltetrazoles (possible for $\lambda \leq 322 \mathrm{~nm}$ ) proceeds best at a wavelength shorter than $295 \mathrm{~nm}$, irrespective of concentration.

\section{INTRODUCTION}

Efficient photochemical synthetic protocols are highly sought-after, whenever spatiotemporal control is desired. Applications, such as 3D laser lithography take advantage of the precise control of photochemical processes by a monochromatic light source. Nevertheless, the most common light sources used in synthetic photochemistry emit a spectrum of wavelengths. While filters can provide irradiation with a relatively narrow incident spectrum, studies of wavelength-dependent photochemistry often lack the precision or versatility of wavelength-tunable lasers. ${ }^{1-8} \mathrm{~A}$ relatively small proportion of phototriggered reactions, such as the intramolecular [5+2] cycloaddition of N-pentenyl dimethylmaleimides, ${ }^{9}$ the isomerization of cytosine, ${ }^{10}$ the photolysis of phenol, ${ }^{11}$ the phototransformation of anethole ${ }^{12}$ and 6-methoxyindole ${ }^{13}$ as well as the photochemical reaction pathways of aminotetrazoles ${ }^{14}$ have been investigated by a monochromatic wavelength assessment. Some of these studies have focused on the photochemistry in an argon matrix or employ a tunable laser as a photodissociation light source in a coupled mass spectrometric setup. ${ }^{15}$ However, only two systematic studies of solution photochemistry at ambient temperature employing tunable laser light have been reported. We recently applied monochromatic tunable laser light to systematically investigate the wavelength-dependent conversion of methyl methacrylate polymerizations initiated by oxime ester photoinitiators. ${ }^{16}$ Furthermore, action plots were reported for the visible-light-induced ligation via pyreneacyl sulfides. ${ }^{17}$ Specifically, lightinduced ligation methods involving cycloadditions have found widespread interest. ${ }^{18-20}$ The Diels-Alder reaction 
between a photochemically generated o-quinodimethane (photoenol) and an electron deficient alkene, ${ }^{21}$ as well as the 1,3-dipolar cycloaddition between a photolytically formed nitrile imine and an alkene (Nitrile Imine mediated Tetrazole Ene Cycloaddition, NITEC) are arguably two of the most important recent examples of highly efficient light-induced ligation reactions. ${ }^{22-26}$

a)
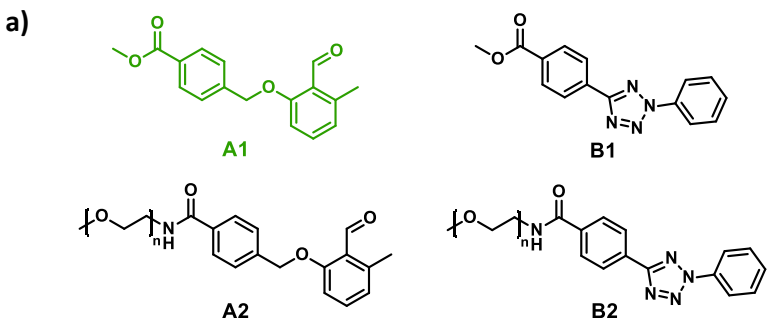

b)
c) 450

B
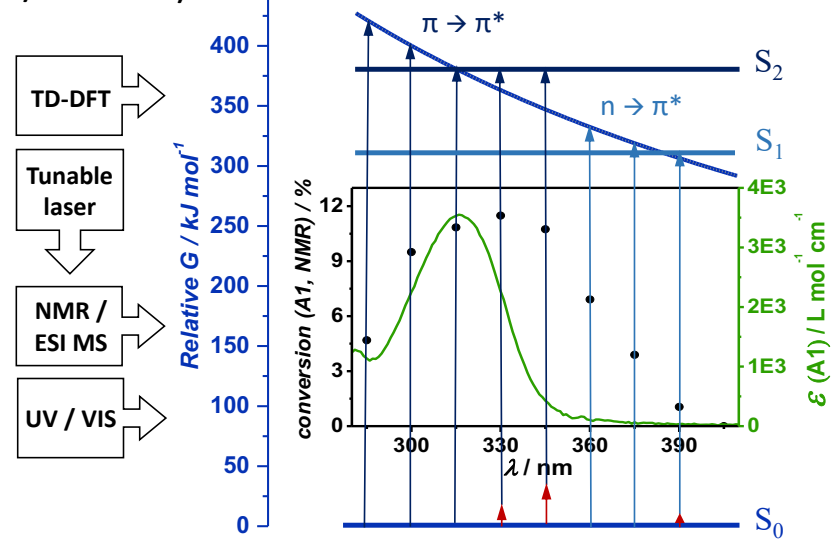

Scheme 1: Schematic overview of the experimental and theoretical approach followed in the current study. (a) Compounds investigated herein. (b) Methodologies: TD-DFT calculations provide energy levels of the excited states for each reaction type; action plots based on monochromatic wavelength scans were determined by NMR or ESI MS and the molar attenuation coefficient was obtained from UV Vis spectra. (c) The relationship between absorptivity, electronic energies and reactivity is explored, enabling the summary of the findings in a Jablonski-inspired diagram. The characteristic excitation energy of each wavelength $\left(E=h c \lambda^{-1}\right.$, blue top curve and blue arrows), combined with contributions of vibrational energy from the ground state (red arrows) leads to specific excited states ( $\mathrm{S}_{2}$ via a $\pi \rightarrow \pi^{*}$ transition and $\mathrm{S}_{1}$ via a $\mathrm{n} \rightarrow \pi^{*}$ transition) and subsequent reactivity (conversion).

Their applications include surface functionalization, ${ }^{27}$ the synthesis of sequence defined macromolecules, 28,29 $\lambda$-orthogonal ligation, ${ }^{30,31}$ light-induced in situ selfassembly of polymers, 32 photoresists design for 3D laser lithography, ${ }^{33}$ synthesis of hydrogels ${ }^{34}$ and functionalization of biomolecules such as labeling of proteins ${ }^{35-38}$ or microRNA. ${ }^{39}$ In addition, nitrile imines are susceptible to reactions with nucleophiles, which was exploited for site selective surface patterning ${ }^{40}$ as well as single chain nanoparticle synthesis. ${ }^{41}$ The bioorthogonality of the nitrile imine mediated tetrazole ene cycloaddition - even though limited in some instances - is of high value for biological applications. ${ }^{42}$
To date, however, the reactivity of tetrazoles and $o$-methylbenzaldehydes has not been mapped as a function of wavelength to provide an encompassing picture of their photochemical behavior. Such an in-depth understanding is essential for their application in the above noted fields and, critically, to enable the targeted design of their photochemistry including highly soughtafter red-shifting making these systems amenable to in vivo chemistry. Herein, we carefully map the reactivity of the compounds (refer to Scheme 1) as a function of wavelength applying an identical photon count. We quantify the effects of absorptivity as well as the energy dissipation after light impact by DFT and multireference calculations to arrive at an encompassing picture of their photochemical reactivity.

\section{RESULTS AND DISCUSSION}

\section{Wavelength dependence of photoenolization}

Previously, $o$-methylarylketones were shown to be activated by UV light and to form a reactive $o$ quinodimethane species, which can subsequently undergo a Diels-Alder reaction with a dienophile. ${ }^{43-45}$ The reaction has been widely adopted as an efficient photoligation. $21,24,46-49$ Herein, we initially probe the conversion of the photoinduced reaction of the $O$ methylbenzaldehyde-containing model compound A1 with N-ethylmaleimide (refer to Figure 1a) in its photoactive range of $285 \mathrm{~nm}$ to $390 \mathrm{~nm}$. A 1.1-fold excess of the dienophile was used to ensure that the transiently generated photoenols are quantitatively trapped. Photoreactions were performed in sealed cylindrical flasks in deoxygenated solutions at ambient temperature (refer to the Supporting Information, chapter 4 and 5 for details). Identical samples were irradiated with monochromatic nanosecond laser pulses (spectral width < $0.3 \mathrm{~nm}, 100 \mathrm{~Hz}$ ) of wavelengths in the range of $285 \mathrm{~nm}$ to $405 \mathrm{~nm}$. Conversion was determined by ${ }^{1} \mathrm{H}-\mathrm{NMR}$ spectroscopy using the resonances A1d, A1Pc and A1Pf (refer to Figure $1 \mathrm{a}-\mathrm{c}$ ).

Inspection of Figure 1c indicates the formation of product A1P at all probed wavelengths except $405 \mathrm{~nm}$. The wavelength dependent conversion, with values between $1 \%$ at $390 \mathrm{~nm}$ and $11.5 \%$ at $330 \mathrm{~nm}$, is depicted in Figure 1c (action plot). Quantum yields were determined numerically by simulation of the photoreaction with spatiotemporal precision using the experimental parameters, molar attenuation coefficients of all absorbing species in the solution and the observed conversion by application of a modified custom written algorithm (refer to the Supporting Information, chapter 7). ${ }^{50}$ Critically, Figure 1c further indicates that the plot of conversion vs. wavelength does not adhere to the absorption spectrum of the reactant. Previously, we observed similar significant deviations between absorptivity and reactivity in photoinitiating systems. ${ }^{16,51}$ The interpretation of these results is only possible by considering - besides the molecular energy dissipation 
mechanisms (see below) - how light is attenuated in each sample solution. Thus, Beer-Lambert's law is employed to calculate the light attenuation as a function of the wavelength for all photochemical ligations reported herein.

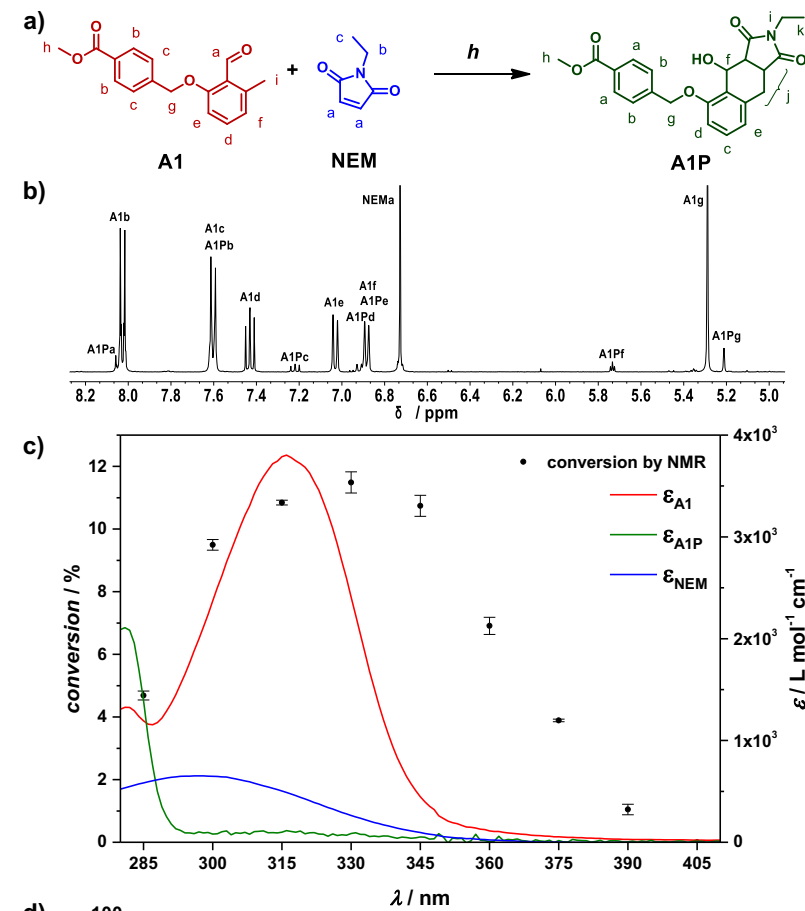

d)

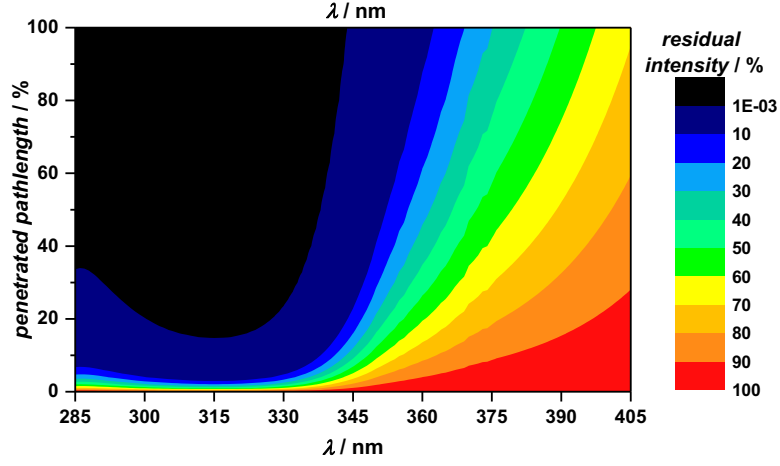

Figure 1: (a-d) Analysis of wavelength dependent reactivity of individual samples of $1.98 \mu \mathrm{mol}\left(7.92 \mathrm{mmol} \mathrm{L}^{-1}\right) \mathbf{A 1}$ with $2.19 \mu \mathrm{mol}$ N-ethylmaleimide (NEM) in $0.25 \mathrm{~mL} \mathrm{CD}{ }_{3} \mathrm{CN}$ using monochromatic light ( 285 - $405 \mathrm{~nm}, 15 \mathrm{~nm}$ steps; $0.30 \pm 0.02$ $\mu$ mol photons; 1000 pulses; pulse frequency $100 \mathrm{~Hz} ; 18{ }^{\circ} \mathrm{C}$ ). (a) Reaction of $\mathbf{A 1}$ and NEM. (b) Expansion of an example NMR spectrum (400 MHz, $\mathrm{CD}_{3} \mathrm{CN}$ ) of a sample irradiated at $315 \mathrm{~nm}$ and resonance assignments. (c) Observed conversion (action plot) and molar attenuation coefficient of A1, NEM and A1P. (d) Light attenuation map: The residual light intensity of the penetrating laser beam (first laser pulse, color coded intensity) in the sample solution containing A1 and NEM is depicted as a function of the wavelength of the incident light. Details of the underpinning Beer-Lambert's law calculations can be found in the Supporting Information.

Figure $1 \mathrm{~d}$ shows the residual light intensity at any point along the axis of the laser beam in the sample solutions during the first laser pulse. The molar attenuation coefficients of the reactants have a strong influence on the penetration depth of the incident beam. Figure 1d suggests that over a broad wavelength range virtually all incident photons are absorbed in only a small part of the solution, while at long wavelengths and thus low molar attenuation coefficients, the beam penetrates further into the solution. It needs to be emphasized that in the case of full absorption of the light in the solution and no competing absorption of other species than the reactant, the conversion should be independent of the wavelength. This is despite a varying molar attenuation coefficient and is because each wavelength is assumed to trigger an equally efficient pathway to the transient species. Consequently, considering absorption effects in the performed experiment, the observed conversion values (refer to Figure 1) can be related to the efficiency of the reaction as a function of the wavelength.

In the following, the relationship between the absorption spectrum and the action plot is discussed with increasing wavelength. At the high energy end of the spectrum, i.e. at $285 \mathrm{~nm}$, conversion is lower in comparison to the samples irradiated with wavelengths 300 $345 \mathrm{~nm}$, although the identical number of photons was applied to, and absorbed by the sample solution. However, the UV Vis spectrum of the Diels-Alder cycloadduct shows a significant absorption at $285 \mathrm{~nm}$, potentially contributing to a lowered conversion due to competing absorption. Yet only a conversion of five percent was observed in the sample, indicating that another process potentially lowers the conversion efficiency. It is hypothesized that excitation into the third singlet excited state $S_{3}$ could lead to parasitic photophysical processes that consume energy without leading to the product.

From the action plot, it can qualitatively be concluded that reactivity is highest and nearly constant in the wavelength range from $300 \mathrm{~nm}$ to $345 \mathrm{~nm}$. Differences within the observed conversion values $(300 \mathrm{~nm}$ to $345 \mathrm{~nm}$ ) are partly due to the absorption of light by Nethylmaleimide. This wavelength range correlates with a broad absorption band $\left(\lambda_{\max }=316 \mathrm{~nm}\right)$, which can be assigned to a $\pi \rightarrow \pi^{*}$ transition into the $S_{2}$ state. Within this wavelength range no significant wavelength dependence of the reactivity is observed, indicating that any excess energy in the $S_{2}$ state is dissipated by vibrational relaxation, leading to the vibrational ground state of $S_{2}$ prior to further processes. At $330 \mathrm{~nm}$, the quantum yield of the reaction was numerically calculated from the observed conversion as $0.93 \pm 0.10$, considering competing absorption of NEM as well as the mean deviation in the conversion and the mean deviation in the measured transmittance of the glass vials used for the tunable laser experiments (refer to the Supporting Information, chapter 5.1 and 7). Already in 1961, Yang and Rivas estimated the quantum yield of the photoenolization of $o$-methylbenzophenone and subsequent trapping with an electron poor dienophile to be higher than $0.5 .{ }^{43}$ The high quantum yield of the reaction reported here is thus not entirely unexpected, as monochromatic $330 \mathrm{~nm}$ irradiation of a second generation 
photoenolizable $o$-methylbenzaldehyde ${ }^{24}$ constitutes an optimized and efficient photoligation.

Further in the longer wavelength regime, from $360 \mathrm{~nm}$ up to $390 \mathrm{~nm}$, the low absorption can be assigned to a symmetry forbidden $n \rightarrow \pi^{*}$ transition into the $S_{1}$ state, potentially competing with a long wavelength tail of the $\pi \rightarrow \pi^{*}$ transition in the case of the wavelengths 360 and $375 \mathrm{~nm}$. Time-dependent density functional theory calculations with the M062X functional predict the $S_{2}$ to $\mathrm{S}_{1}$ gap to be $75 \mathrm{~kJ} \mathrm{~mol}^{-1}$. This supports the explanation that reactivity at these wavelengths is due to excitation into the $S_{1}$ state. Considering the light attenuation map shown in Figure 1d, it is concluded that, in the case of $390 \mathrm{~nm}$ irradiation, half of the incident photons are absorbed by the sample solution initially. With only one percent conversion in this sample, a quantum yield of $0.15 \pm 0.04$ is calculated numerically. The quantum yield plotted against wavelength is included in the Supporting Information, chapter 7.

The significant decrease of efficiency that occurs from the wavelength $345 \mathrm{~nm}$ to $390 \mathrm{~nm}$ can be rationalized mainly by two pathways. On the one hand, relaxation from $S_{2}$ after internal conversion to $S_{1}$ may proceed along a different geometric pathway in comparison to relaxation after direct excitation into $S_{1}$. The rate of radiationless deactivation consequently depends on the geometry that the excited molecule is in. ISC is the highly preferred pathway compared to radiationless deactivation and the quantum efficiency of the reaction is thus higher, when absorption is caused by the $\pi \rightarrow \pi^{*}$ transition in contrast to the $\mathrm{n} \rightarrow \pi^{*}$ transition. A similar observation was made experimentally with femtosecond time-resolved photoelectron spectroscopy in the case of the excitation of styrene..$^{52}$ On the other hand, ISC from $\mathrm{S}_{1}$ would presumably occur significantly more rapidly, if the molecule carries excess energy, either gained from excitation into high vibrational levels of $S_{1}$ or from the excess energy that is freed after internal conversion from the $S_{2}$ state. Excitation into the Franck-Condon point rather than into the equilibrium excited state may lead to a comparably more efficient ISC and thus quantum yield.

Since the experimental determination of wavelengthdependent conversions only affords information regarding directly excited states, theoretical calculations were carried out to map the energies of all relevant electronic and transition states both for the photochemical mechanism as well as a hypothetical thermal pathway of the reaction. Relative energy levels of excited and transition states, calculated with time-dependent density functional theory are depicted in Figure 2 . Without photochemical activation, the barrier of both intramolecular $\mathrm{H}$-transfer and isomerization of photoenol species is insurmountable at ambient temperature. The observed full retention of $\mathbf{A 1}$ under irradiation with $405 \mathrm{~nm}$ light shows that the reaction only proceeds when the sample is irradiated with light of a suitable wavelength. Here, the mechanism of the processes following intersystem crossing is discussed based on theoretical calculations. An intramolecular [1,5]-H-shift, exhibiting a low activation barrier, first exclusively leads to the (E)-photoenol, which can subsequently isomerize to the (Z)-photoenol with a similarly low activation barrier. We found that the triplet and singlet surface appear to be close at the transition state of the photoenol isomerization, indicating a potential conical intersection. For similar enol molecules, conical intersections at transition states of the enol isomerization were proposed and investigated theoretically. ${ }^{53}$ Thus, we studied the relative energy of the triplet and singlet surfaces of (E)- and (Z)-photoenol during rotation of the respective $\mathrm{C}-\mathrm{C}$ bond in detail using complete active space (CAS) calculations (refer to Figure 3). The results strongly indicate that the triplet (E)-photoenol can be transformed via a conical intersection during rotation to the singlet (Z)-photoenol. The process is almost barrier free, highly efficient and potentially a very selective pathway towards the singlet (Z)-photoenol. This species cannot undergo re-isomerization due to the double bond character of the diene and consequently gets trapped by NEM in the Diels-Alder reaction.

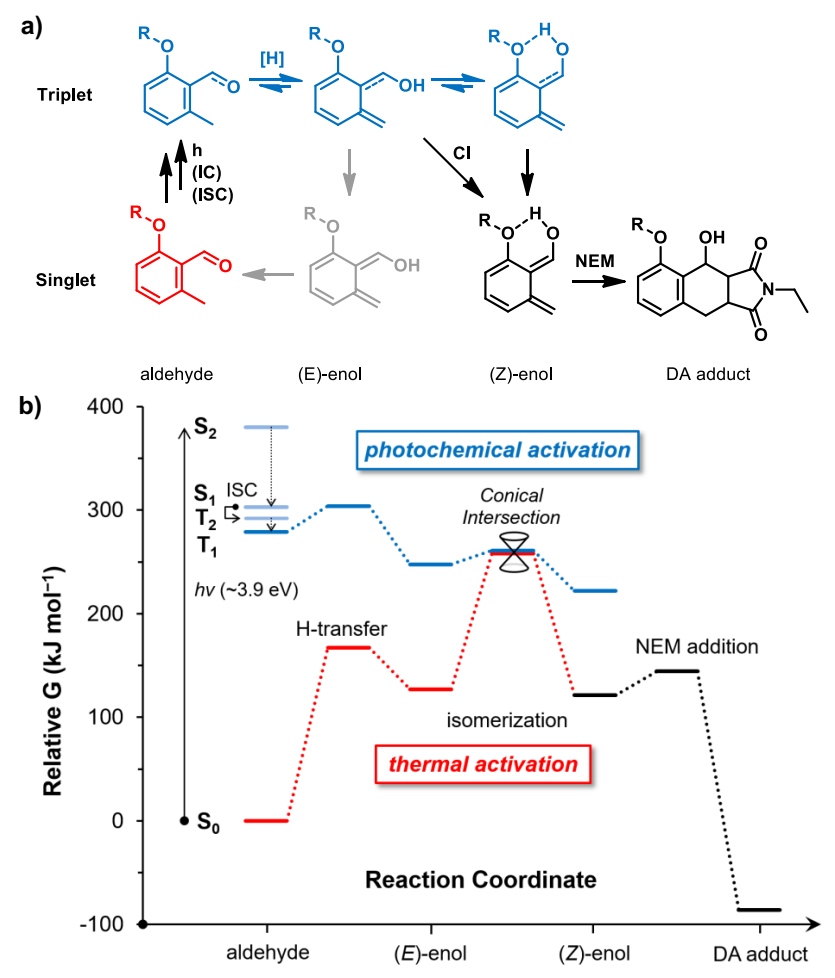

Figure 2: (a) Mechanism of the reaction of A1 with NEM. The species (triplet - blue, singlet - red and black) relate to the diagram below. (b) Relative energy levels for the photochemical (blue) and thermal (red) reaction pathway, calculated with G3(MP2)RAD//M06-2X/6-31+G(d,p). Relevant energy levels are shown for the excitation, intramolecular H-transfer to yield the (E)-photoenol species, isomerization between (E) and (Z)-photoenol via a conical intersection (CI) at the transition state and subsequent Diels-Alder reaction of the (Z)photoenol with NEM. 
a)

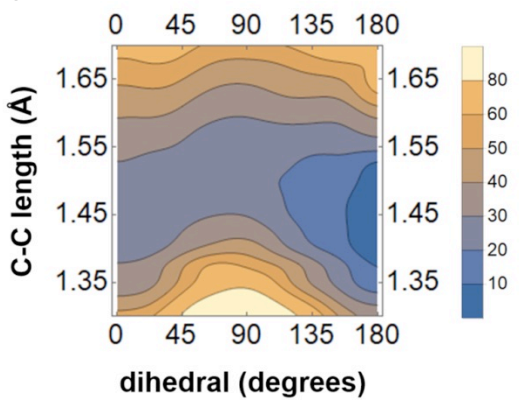

b)

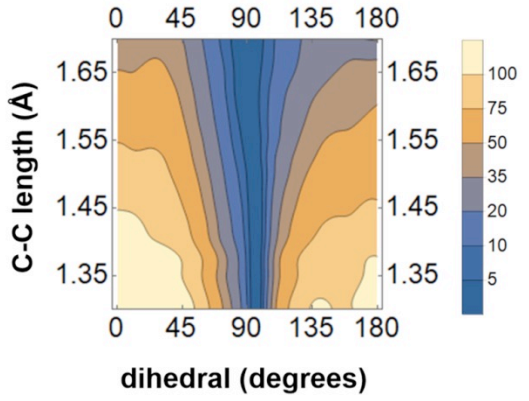

c)

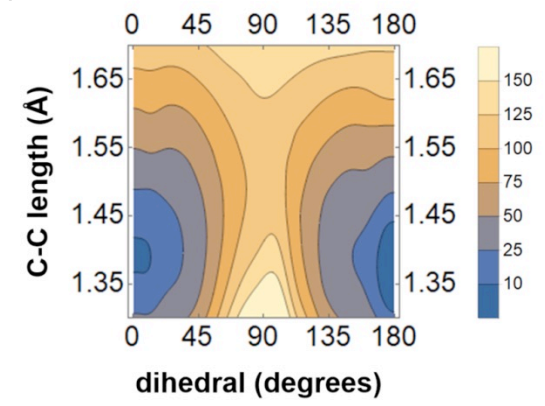

Figure 3: (a) Relative SA-CASSCF electronic energies of the $\mathrm{T}_{1}$ surface (kJ mol-1) of a model (E)- and (Z)-photoenol, plotted as a function of the respective $\mathrm{C}-\mathrm{C}$ dihedral angle and bond length. (b) The $\mathrm{S}_{0}-\mathrm{T}_{1}$ energy gap $\left(\mathrm{kJ}^{-} \mathrm{mol}^{-1}\right)$ over the same region is depicted. A conical intersection seam is clearly visible in the C-C dihedral angle range of $90-100^{\circ}$. (c) Relative SA-CASSCF electronic energies of the $\mathrm{S}_{0}$ surface $\left(\mathrm{kJ} \mathrm{mol}^{-1}\right)$ of the model (E)- and (Z)-photoenol plotted over the same region.

While the action plot of $\mathbf{A 1}$ (refer to Figure 1c and 1d) was obtained with near complete absorption of photons in the solution, conditions under which the sample solution is more thoroughly penetrated can be a valuable model for photo-flow chemical synthesis. Yet, analysis of the conversion by NMR is challenging for very low sample concentrations. The amount of required substrate can be reduced drastically by employing high resolution electrospray ionization mass spectrometry for the relative quantitative analysis of well-ionizing macromolecular samples. $O$-methyl-benzaldehyde terminal poly(ethylene glycol) (PEG) A2 was prepared to qualitatively determine the wavelength dependent efficiency of the reaction at a concentration of $0.28 \mathrm{mmol} \mathrm{L}$ 1 (28-fold dilution compared to the previous experiments employing A1, refer to Figure 2 and 3). A 4.2-fold excess of NEM was employed to facilitate the trapping of the transient species at the lowered concentration. The progress of the reaction was traced by high resolution ESI MS (refer to Figure 4 and the Supporting Information, chapter 5.2 , for details).

We have previously shown that mass spectra of macromolecules with a well ionizing lateral chain such as poly(meth)acrylates ${ }^{51,54-57}$ can be employed for quantitative analysis. In the field of proteomics, a range of tools also exist for the relative quantitative evaluation of peptide mass spectra. ${ }^{58}$ A sophisticated evaluation procedure (refer to the Supporting Information, chapter 6 ) is employed here to reduce potential ionization biases. By applying an identical analysis procedure to each sample, the obtained (apparent) mole fractions - regardless of a potential systematic error - are a measure of the efficiency of the reaction. Although formation of the product was observed in samples irradiated with light in the wavelength range of $285 \mathrm{~nm}$ to $390 \mathrm{~nm}$, the action spectrum appears to follow the absorbance of the $o$-methylbenzaldehyde moiety closely. The light attenuation map of the irradiation of the samples at the low concentration (refer to Figure 2d) reveals that within the wavelength range of $360 \mathrm{~nm}$ to $405 \mathrm{~nm}$ initially more than $90 \%$ of the incident light passes through the solution. Consequently, only a fraction of the photons of these wavelengths are absorbed and can trigger the reaction, leading to a strongly decreased conversion relative to the samples irradiated with light of shorter wavelengths.

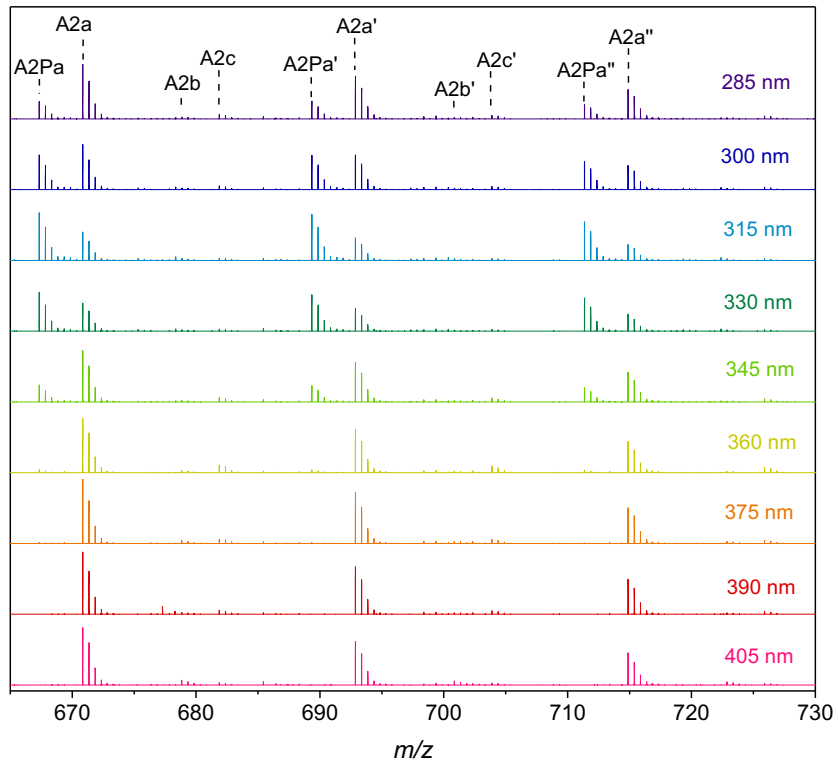

Figure 4: Example mass spectra of samples irradiated in the wavelength range of $285-405 \mathrm{~nm}$ (see Figure 5 and the Supporting Information, chapter 5.2 for details and ion assignments).

With no complete absorption of the beam at any wavelength, the conversion plot necessarily resembles the absorption plot to a first approximation. The two wavelength-dependent conversion plots shown in Figure 1c and $5 \mathrm{c}$ have a different shape based on how light is absorbed in the respective sample solutions and are not in contradiction to each other. Importantly, only in the case of strong absorption of the incident beam can statements be made about the wavelength dependent efficiency of photoreactions. We conclude that on a practical level, the absorption spectrum can be used as a wavelength selection guide only for experiments that 
are conducted with deep penetration of the solution with light. On the experimental level, this can be the case for photo-flow reactions. In contrast, in batch experiments even a weakly allowed $n \rightarrow \pi^{*}$ transition can become a significant pathway, allowing the efficient use of longer wavelengths.
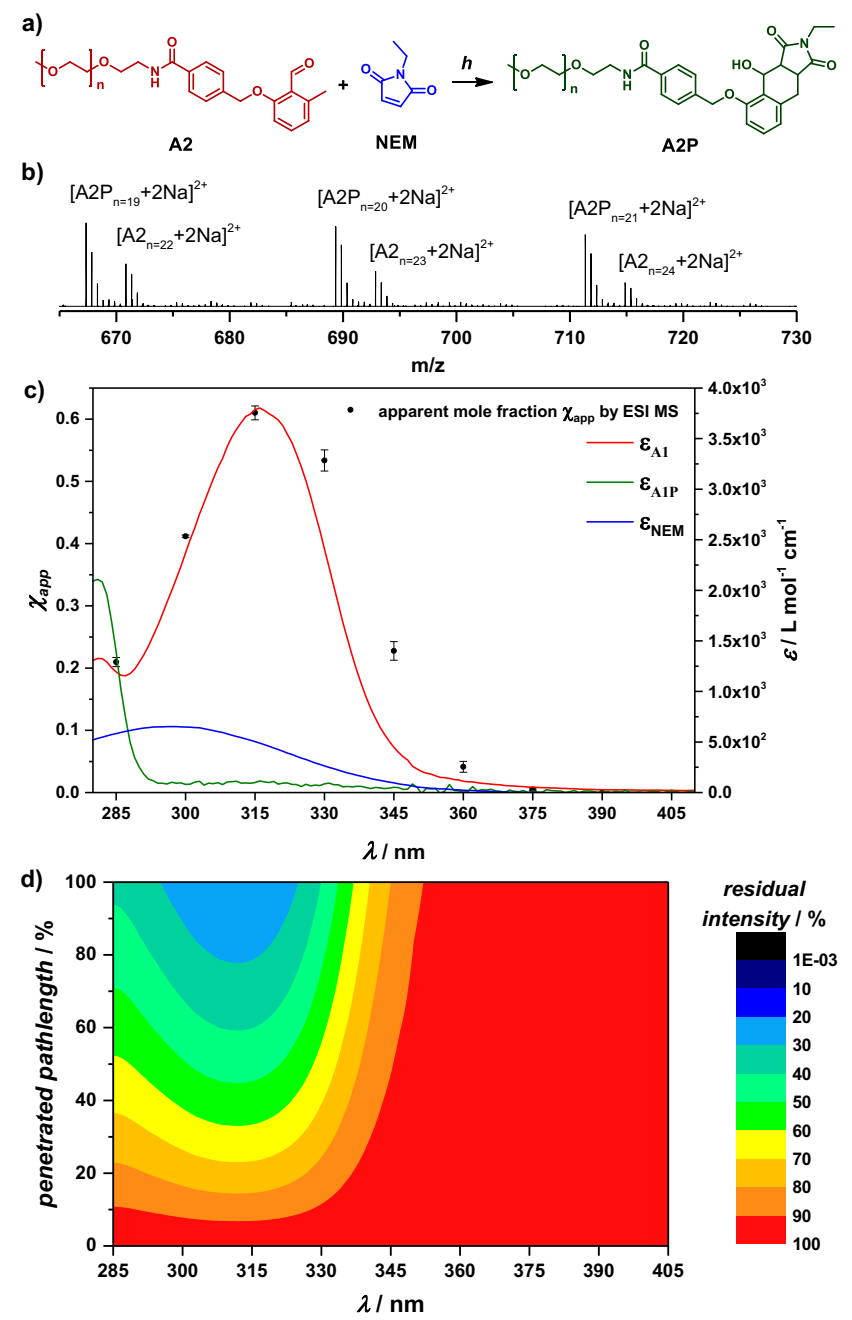

Figure 5: (a-d) Analysis of wavelength-dependent reactivity of samples each containing $0.028 \mu \mathrm{mol}\left(0.28 \mathrm{mmol} \mathrm{L}^{-1}\right) \mathrm{A2}$ and $0.118 \mu \mathrm{mol}$ NEM in $0.1 \mathrm{~mL} \mathrm{CD}{ }_{3} \mathrm{CN}$ using monochromatic light (285 - $405 \mathrm{~nm}, 15 \mathrm{~nm}$ steps; $30 \pm 2$ nmol photons; 100 pulses; pulse frequency $100 \mathrm{~Hz} ; 18{ }^{\circ} \mathrm{C}$ ). (a) Reaction of $\mathbf{A} 2$ and NEM. (b) Expansion of an example high-resolution mass spectrum of the sample irradiated at $315 \mathrm{~nm}$. (c) Observed apparent mole fraction $\chi_{\text {app }}$ by ESI MS (action plot, $\chi_{\text {app }}=\mathrm{I}_{\mathbf{A} 2 \mathbf{P}} \mathrm{I}_{\mathbf{A} 2^{-1}} \mathrm{I}_{\mathbf{A} 2 \mathbf{P}^{-1}}$ ) and molar attenuation coefficient of A1, NEM and A1P. (d) Light attenuation map: Residual light intensity of the penetrating laser beam (first laser pulse, color coded intensity) in a sample solution containing A2 and NEM is depicted as a function of the wavelength of the incident light.

Considering the ongoing quest for visible light activatable functionalities, this observation underpins the significance of theoretical calculations of excited state energies of proposed red-shifted $o$-methylarylaldehyde species.

\section{Wavelength dependence of nitrile imine mediated tetrazole ene cycloaddition}

By analogy to the photoenol-based experiments the small molecule 2,5-diphenyltetrazole model compound B1 and the polymer bound analogue B2 were synthesized and subjected to a wavelength dependent constant photon count assessment.

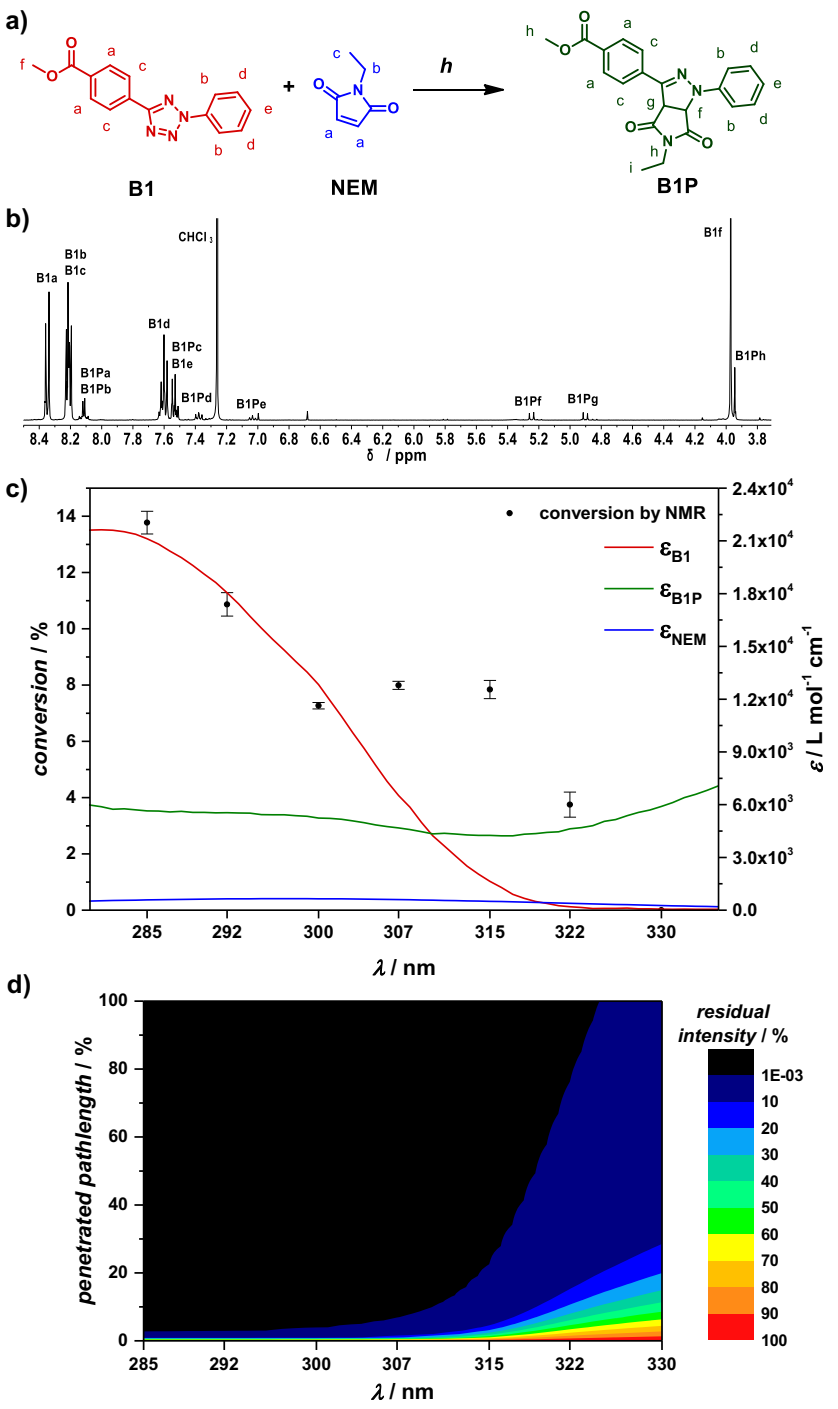

Figure 6: (a-d) Analysis of wavelength-dependent reactivity of samples each containing $2.40 \mu \mathrm{mol}\left(9.6 \mathrm{mmol} \mathrm{L}^{-1}\right) \mathrm{B1}$ and $2.70 \mu \mathrm{mol} N \mathrm{NEM}$ in $0.25 \mathrm{~mL} \mathrm{CD}{ }_{3} \mathrm{CN} / \mathrm{C}_{6} \mathrm{D}_{6} 1: 1(\mathrm{v} / \mathrm{v})$ using monochromatic laser light $(285-330 \mathrm{~nm}, 7$ and $8 \mathrm{~nm}$ steps; $0.6 \pm 0.04 \mu \mathrm{mol}$ photons; 2000 pulses; pulse frequency 100 $\mathrm{Hz} ; 18{ }^{\circ} \mathrm{C}$ ). (a) Reaction of $\mathbf{B 1}$ and NEM. (b) Expansion of a typical ${ }^{1} \mathrm{H}-\mathrm{NMR}$ spectrum $\left(400 \mathrm{MHz}, \mathrm{CDCl}_{3}\right)$ of the sample irradiated at $285 \mathrm{~nm}$. (c) Observed conversion (action spectrum) and molar attenuation coefficient of B1, NEM and B1P. (d) Light attenuation map: The residual light intensity of the penetrating laser beam (first laser pulse, color coded intensity) in the sample solution containing B1 and NEM is depicted as a function of the wavelength of the incident light.

The wavelengths were adjusted to the range of $285 \mathrm{~nm}$ to $330 \mathrm{~nm}$, as reactivity was only observed up to 322 $\mathrm{nm}$. Conversion of B1 with NEM to B1P was determined 
by ${ }^{1} \mathrm{H}-\mathrm{NMR}$ spectroscopy (via the resonance B1a-c, B1Pa, B1Pb and B1Pd-g, refer to Figure 6b) after irradiation of samples drawn from a stock solution of B1 and NEM in a 1:1 mixture of benzene and acetonitrile (refer to Figure $6 \mathrm{a}-\mathrm{c}$ ). The solvent mixture was selected to dissolve both reactants and products as well as to ensure that no competing absorption of the incident light by the solvent occurs.

As observed for the $o$-methylbenzaldehyde species A1, conversion of the small molecular tetrazole B1 does not adhere to the respective absorbance (refer to Figure $6 \mathrm{c}$ ). With full absorption of the laser beam in the solutions at all wavelengths (refer to Figure 6d) and a similar degree of absorption due to the pyrazoline product at the varied wavelengths, the action plot depicted in Figure $6 \mathrm{c}$ can be employed to estimate the efficiency of the involved photochemical processes qualitatively. Interpretation of the results is more challenging than in the case of the photoenolization, as the product of the photoreaction, a pyrazoline, absorbs photons at every wavelength under consideration. While the reaction progresses, the formation of product causes competing absorption, which is more relevant for larger ratios of the molar attenuation coefficient of the pyrazoline product to the tetrazole reactant. As light in the solution is strongly attenuated as calculated with Beer Lambert's law, diffusion of the formed product from a volume with high light intensity to the dark volume segments decreases the effect of competing absorption of the product at low conversion values.

The mechanism and relative energies of all involved transition states can assist in the interpretation of the results. Thus, a potential correlation of the experimentally observed conversion and theoretically calculated energy levels of excited states ${ }^{59}$ (refer also to Figure 7) is discussed in the following with increasing wavelength. Irradiation of a solution of $2.4 \mu \mathrm{mol} \mathbf{B 1}$ and 2.7 $\mu \mathrm{mol}$ NEM with $0.6 \pm 0.04 \mu \mathrm{mol}$ photons (refer to Figure 5) can theoretically yield not more than $25 \%$ conversion. Further, an unknown fraction of the incident photons is, over the course of the experiment, absorbed by the product of the reaction or NEM. Thus, the observed conversion values after excitation with $285 \mathrm{~nm}$ and $292 \mathrm{~nm}$ light evidence that the NITEC reaction proceeds with a remarkably high efficiency in this wavelength regime, the minimum quantum yield at $285 \mathrm{~nm}$ is estimated as $0.55 \pm 0.06$. a)
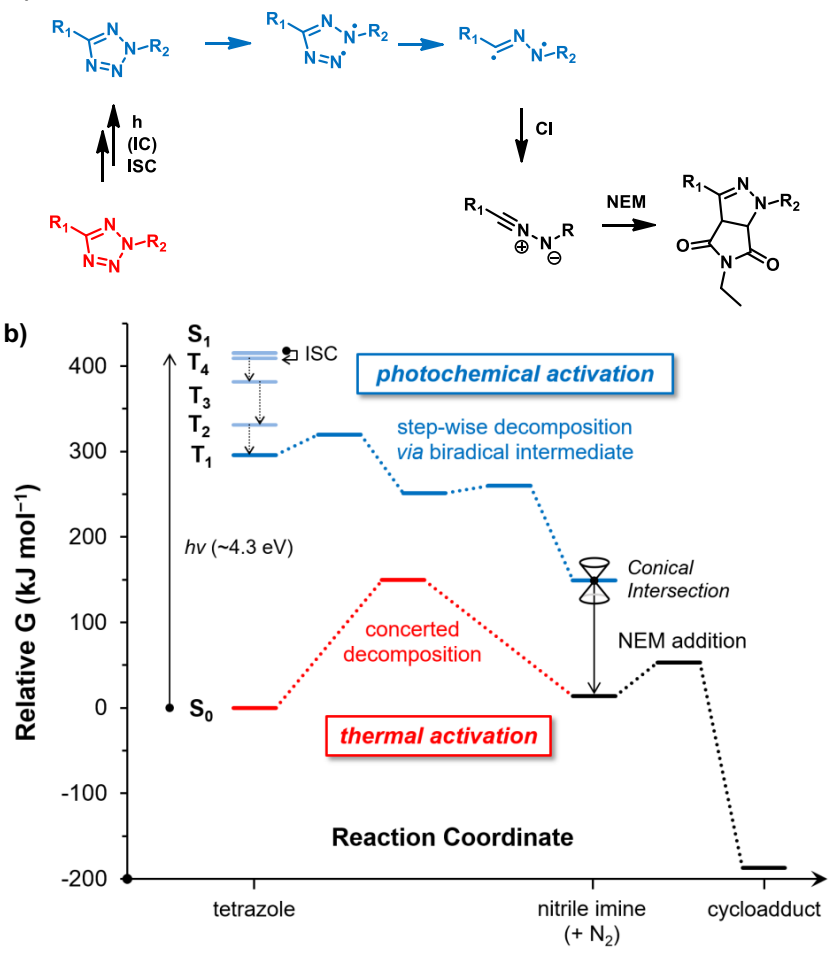

Figure 7: (a) Mechanism of the reaction of B1 with NEM. Triplet species are highlighted in blue, while singlet species are shown in red and black. (b) Relative energy levels for the photochemical (blue) and thermal (red) reaction pathway, calculated with TD-DFT and G3(MP2)RAD/M06-2X/6$31+G(d, p)$. Relevant energy levels are shown for the excitation, decomposition to the nitrile imine species and the 1,3dipolar cycloaddition with NEM.

A correlation with an excitation into the $\mathrm{S}_{1}$ state with a potentially very efficient intersystem crossing into the $\mathrm{T}_{3}$ state, which was previously estimated as having a relative energy of $\Delta \mathrm{GT}_{\mathrm{T} 3}=406 \mathrm{~kJ} \mathrm{~mol}^{-1}(4.21 \mathrm{eV}$, $295 \mathrm{~nm}),{ }^{59}$ is proposed here. We reported that the tetrazole $\mathbf{B 1}$ has a vertical $S_{1}$ state close to the $T_{3}$ state and can thus be expected to exhibit a fast ISC. Excitation into lower vibrational levels of $\mathrm{S}_{1}$, which could lead by a presumably slower ISC to the $\mathrm{T}_{2}$ or $\mathrm{T}_{1}$ state potentially correlates with a lowered conversion at wavelengths longer than $295 \mathrm{~nm}$. The relative energy of the optimized $\mathrm{S}_{1}$ state was reported earlier as $\Delta \mathrm{G}_{\mathrm{s} 1 \text {, opt }}=384 \mathrm{~kJ}$ mol-1 $^{-1}(3.98 \mathrm{eV}, 312 \mathrm{~nm}){ }^{59}$ Reactivity at $322 \mathrm{~nm}$, relating to an activation of the tetrazole with photons not carrying sufficient energy to excite into the $S_{1}$, according to aforementioned quantum chemical calculations, can thus be explained by a vibrational energy contribution to the excitation. Given that the triplet state is a crucial intermediate in the NITEC reaction, ${ }^{59}$ further quantum chemical calculations were performed with the aim to arrive at a complete picture of the reaction path (refer to Figure 7). 


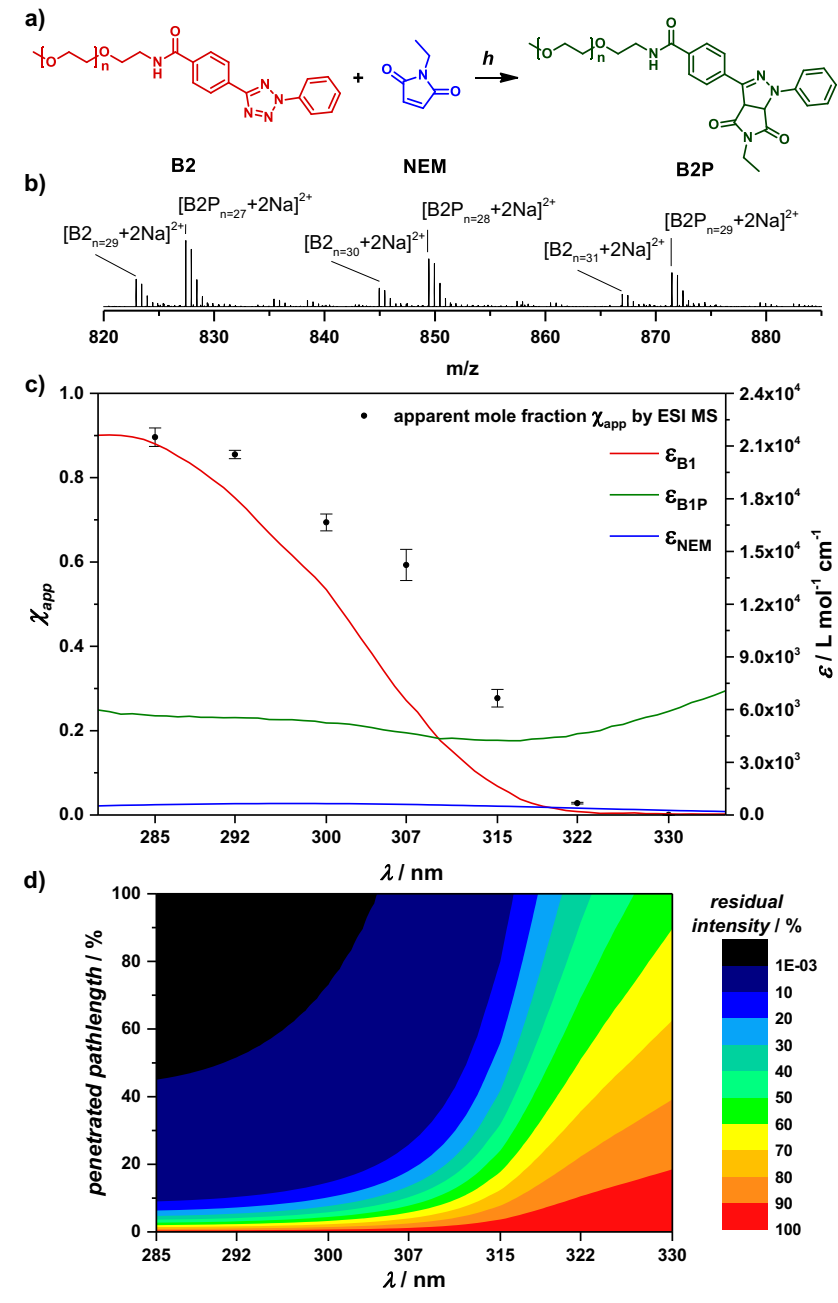

Figure 8: (a-d) Analysis of wavelength-dependent reactivity of samples each containing $0.12 \mu \mathrm{mol}\left(1.2 \mathrm{mmol} \mathrm{L}^{-1}\right) \mathbf{B} 2$ with $0.20 \mu \mathrm{mol}$ NEM in $0.1 \mathrm{~mL} \mathrm{CH}_{3} \mathrm{CN}$ using monochromatic laser light (285 - $330 \mathrm{~nm}, 7$ and $8 \mathrm{~nm}$ steps; $60 \pm 4 \mathrm{nmol}$ photons; 200 pulses; pulse frequency $100 \mathrm{~Hz} ; 18^{\circ} \mathrm{C}$ ). (a) Reaction of $\mathbf{B 2}$ and NEM. (b) Expansion of a typical high-resolution mass spectrum of the sample irradiated at $300 \mathrm{~nm}$. (c) Observed

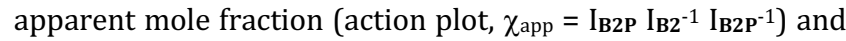
molar attenuation coefficient of B1, NEM and B1P. (d) Light attenuation map: The residual light intensity of the penetrating laser beam (first laser pulse, color coded intensity) in the sample solution containing B2 and NEM is depicted as a function of the wavelength of the incident light.

The thermal activation, known to proceed only at temperatures exceeding $150^{\circ} \mathrm{C},{ }^{60}$ was calculated to proceed via a concerted decomposition with a barrier of $150 \mathrm{~kJ}$ mol $^{-1}$. Excitation leads to a singlet excited state, which subsequently undergoes ISC to a triplet state. On the triplet surface, a step-wise decomposition is possible with low barriers via a diradical intermediate. Preliminary calculations indicate that the nitrile imine can relax from its $\mathrm{T}_{1}$ to $\mathrm{S}_{0}$ state via a conical intersection, through straightening of the core $\mathrm{C}-\mathrm{N}-\mathrm{N}$ group. The formed singlet species is trapped in the rapid 1,3-dipolar cycloaddition with NEM.

While relative energies of excited states could be derived from action spectra in case of the $o$-methylbenzaldehyde activation, such an approach is not directly possible for the NITEC reaction due to the competing absorption effects of the pyrazoline product. Due to Beer-Lambert's law a closer correlation between absorptivity and reactivity of the activatable group in experiments with a deeper light penetration should be expected, although the absorption due to the pyrazoline potentially affects the action spectrum. Thus, in analogy to the previous experiments employing the $o$-methylbenzaldehyde-terminal PEG A2, a 2,5-diphenyltetrazole-terminal PEG B2 was prepared and investigated (refer to Figure 8 a-d). Apparent mole fractions were obtained by evaluation of high resolution electrospray ionization mass spectra (refer to the Supporting Information, chapter 5.4 and 6). The obtained action spectrum is discussed as a qualitative measure of the NITEC efficiency at varied wavelengths. The associated action plot mirrors the absorbance of the tetrazole moiety more closely than in the experiments conducted at higher concentration, emphasizing the influence of absorption effects at lowered concentrations (1.2 $\left.\mathrm{mmol} \mathrm{L}^{-1} v s .9 .6 \mathrm{mmol} \mathrm{L}^{-1}\right)$.

The observed relationship between absorbance and action spectrum at good light penetration appears to be valid to a first approximation for both light induced ligations. With this largely being an absorption effect, a relation of energy levels of electronic excited states with the wavelength dependent conversion can only be established within reasonably strong absorbing solutions. In the case of the NITEC reaction, the action spectrum (refer to Figure 6c) supports previously reported quantum chemical calculations and the significance of an efficient intersystem crossing from the excited singlet state to the triplet surface. A practical implication is that 2,5-diphenyltetrazoles perform best at a wavelength below $295 \mathrm{~nm}$, but can be employed for photoligation up to a wavelength of $322 \mathrm{~nm}$.

\section{CONCLUSIONS}

We quantify the wavelength dependence of two key photochemical ligation protocols, i.e. the photoenolization of $o$-methylbenzaldehydes and the photolysis of tetrazoles using a sophisticated wavelength-tunable laser system. The reactions are found to proceed with remarkably high efficiency after excitation with suitable wavelengths. Correlations between the absorptivity of the reactive moieties, their electronic properties and reactivity are probed, leading to an in-depth understanding of their wavelength-dependent reactivity and mechanism. $O$-methylbenzaldehydes can be excited into the $S_{2}$ by a $\pi \rightarrow \pi^{*}$ transition ( $300 \mathrm{~nm} \leq \lambda \leq 345 \mathrm{~nm}$ ), or the $S_{1}$ by an $n \rightarrow \pi^{*}$ transition (360 $\mathrm{nm} \leq \lambda \leq 390 \mathrm{~nm}$ ), with reactivity in the latter wavelength range being strongly wavelength-dependent in contrast to the $\pi \rightarrow$ $\pi^{*}$ transition. By ISC a triplet $o$-methylbenzaldehyde is formed, which rearranges into the triplet (E)-enol. Through a conical intersection at the transition state of 
the (E)-(Z) isomerization, the singlet (Z)-photoenol is efficiently formed, allowing the Diels-Alder cycloaddition to proceed. The highest efficiency of the NITEC reaction was observed at wavelengths close to the maximum of the tetrazole absorption, correlating with an excitation into $S_{1}$ with subsequent ISC to $T_{3}$. Detailed information regarding the wavelength dependence of photoreactions reported here aids in the design of $\lambda$ orthogonal ligation systems ${ }^{31}$ and advanced photoligation protocols. Consequently, a flow chemistry setup that guarantees full penetration of the passing solution with the incident light is ideally equipped with a light emission source that has an emission maximum of $\lambda=$ $315 \mathrm{~nm}$ in case of the $o$-methylbenzaldehydes reported here and an emission maximum of $\lambda \leq 295 \mathrm{~nm}$ in case of 2,5-diphenyltetrazoles. In contrast, suitable wavelengths for irradiation of batch experiments on a preparative scale are performed with the highest efficiency of photoenolization of $o$-methylbenzaldehydes in the wavelength range of $300 \mathrm{~nm}$ to $345 \mathrm{~nm}$. A weakly allowed $\mathrm{n} \rightarrow \pi^{*}$ transition can be used to design $\lambda$ orthogonal systems involving activation of $o$ methylbenzaldehydes at wavelengths up to $390 \mathrm{~nm}$, although efficiency of the reaction is decreased. Establishing the wavelength dependent efficiency of two key rapid light-induced cycloaddition reactions with control of irradiation geometry, wavelength and photon count is critical for developing and tailoring photoreactive materials such as advanced photoresists. ${ }^{61-63}$ Access to precision photochemical orthogonal ligation systems is of critical importance for enabling the fabrication of multifunctional spatially resolved surfaces, for example by microarray printing and subsequent photopatterning of biomolecules on hydrogels. ${ }^{64}$

\section{ASSOCIATED CONTENT}

Supporting information. This material is available free of charge via the Internet at http://pubs.acs.org. It contains information about materials, characterization, synthesis, tunable laser experiments, photoinduced reactions, numerical evaluation of polymer mass spectra, numerical determination of quantum yields and theoretical methodology.

\section{AUTHOR INFORMATION}

\section{Corresponding Author}

*christopher.barnerkowollik@qut.edu.au

*j.blinco@qut.edu.au

*michelle.coote@anu.edu.au

\section{Author Contributions}

All authors have given approval to the final version of the manuscript.

\section{Funding Sources}

Queensland University of Technology (QUT), Australian Research Council (ARC), Helmholtz Association, German Research Council (DFG)

\section{ACKNOWLEDGMENT}

C.B-K. acknowledges key continued support by the Queensland University of Technology (QUT), the Australian Research Council (ARC) in the form of a Laureate Fellowship as well as by the Karlsruhe Institute of Technology in the context of the STN program of the Helmholtz association. M.L.C. acknowledges financial support from the ARC as well as generous allocations of supercomputing time on the National Facility of the Australian National Computational Infrastructure. J.P.M. acknowledges funding of PhD studies by the Queensland University of Technology (QUT).

\section{ABBREVIATIONS}

$\mathrm{CI}$, conical intersection; ESI MS, electrospray ionization mass spectrometry; ISC, intersystem crossing, LASER, Light amplification by stimulated emission of radiation; NEM, Nethylmaleimide; NITEC, nitrile imine mediated tetrazole ene cycloaddition, NMR, nuclear magnetic resonance; PEG, poly(ethylene glycol); RNA, ribonucleic acid; TD-DFT, time dependent density functional theory; UV, ultraviolet.

\section{REFERENCES}

(1) Pozdnyakov, I. P.; Zhang, X.; Maksimova, T. A.; Yanshole, V. V.; Wu, F.; Grivin, V. P.; Plyusnin, V. F. J. Photochem. Photobiol. A 2014, 274, 117.

(2) Bogdanova, A.; Popik, V. V. J. Am. Chem. Soc. 2003, 125, 14153.

(3) Caselli, M.; Ponterini, G.; Vignali, M. J. Photochem. Photobiol. A 2001, 138, 129.

(4) Dauben, W. G.; Hecht, S. J. Org. Chem. 1998, 63, 6102.

(5) Jakúbek, V.; Lees, A. J. Inorg. Chem. 2000, 39, 5779.

(6) McDonagh, A. F.; Agati, G.; Lightner, D. A. Monatsh. Chem. 1998, 129, 649.

(7) Shaw, L. E.; Langford, C. H. Inorg. Chem. 2000, 39, 541.

(8) Morrison, H.; Bernasconi, C.; Pandey, G. Photochem. Photobiol. 1984, 40, 549.

(9) Davies, D. M. E.; Murray, C.; Berry, M.; Orr-Ewing, A. J.; Booker-Milburn, K. I. J. Org. Chem. 2007, 72, 1449.

(10) Lapinski, L.; Reva, I.; Nowak, M. J.; Fausto, R. PCCP 2011, 13,9676 .

(11) Giuliano, B. M.; Reva, I.; Lapinski, L.; Fausto, R. J. Chem. Phys 2012, 136, 024505.

(12) Krupa, J.; Wierzejewska, M.; Nunes, C. M.; Fausto, R. J. Chem. Phys 2014, 140, 105102.

(13) Lopes Jesus, A. J.; Reva, I.; Fausto, R. J. Photochem. Photobiol. A 2017, 336, 123.

(14) Ismael, A.; Fausto, R.; Cristiano, M. L. S. J. Org. Chem. 2016, $81,11656$.

(15) Herburger, A.; van der Linde, C.; Beyer, M. K. PCCP 2017, 19, 10786.

(16) Fast, D. E.; Lauer, A.; Menzel, J. P.; Kelterer, A.-M.; Gescheidt, G.; Barner-Kowollik, C. Macromolecules 2017, 50, 1815.

(17) Tuten, B. T.; Menzel, J. P.; Pahnke, K.; Blinco, J. P.; Barner-Kowollik, C. Chem. Commun. 2017, 53, 4501.

(18) Poloukhtine, A. A.; Mbua, N. E.; Wolfert, M. A.; Boons, G.J.; Popik, V. V. J. Am. Chem. Soc. 2009, 131, 15769.

(19) Arumugam, S.; Popik, V. V. J. Am. Chem. Soc. 2011, 133, 5573.

(20) Sun, L.; Ding, J.; Xing, W.; Gai, Y.; Sheng, J.; Zeng, D. Bioconjugate Chem. 2016, 27, 1200.

(21) Gruendling, T.; Oehlenschlaeger, K. K.; Frick, E.; Glassner, M.; Schmid, C.; Barner-Kowollik, C. Macromol. Rapid Commun. 2011, 32, 807.

(22) Song, W.; Wang, Y.; Qu, J.; Madden, M. M.; Lin, Q. Angew. Chem. Int. Ed. 2008, 47, 2832.

(23) Herner, A.; Lin, Q. Top. Curr. Chem. 2015, 374, 1. 
(24) Pauloehrl, T.; Delaittre, G.; Winkler, V.; Welle, A.; Bruns, M.; Börner, H. G.; Greiner, A. M.; Bastmeyer, M.; BarnerKowollik, C. Angew. Chem. Int. Ed. 2012, 51, 1071.

(25) Ramil, C. P.; Lin, Q. Curr. Opin. Chem. Biol. 2014, 21, 89.

(26) An, P.; Yu, Z.; Lin, Q. Org. Lett. 2013, 15, 5496.

(27) Delaittre, G.; Goldmann, A. S.; Mueller, J. O.; BarnerKowollik, C. Angew. Chem. Int. Ed. 2015, 54, 11388.

(28) Zydziak, N.; Feist, F.; Huber, B.; Mueller, J. O.; BarnerKowollik, C. Chem. Commun. 2015, 51, 1799.

(29) Zydziak, N.; Konrad, W.; Feist, F.; Afonin, S.; Weidner, S.; Barner-Kowollik, C. Nat. Commun. 2016, 7, 13672.

(30) Hiltebrandt, K.; Kaupp, M.; Molle, E.; Menzel, J. P.; Blinco, J. P.; Barner-Kowollik, C. Chem. Commun. 2016, 52, 9426.

(31) Hiltebrandt, K.; Pauloehrl, T.; Blinco, J. P.; Linkert, K.; Börner, H. G.; Barner-Kowollik, C. Angew. Chem. Int. Ed. 2015, 54,2838

(32) Chen, L.; Xu, M.; Hu, J.; Yan, Q. Macromolecules 2017, 50, 4276

(33) Quick, A. S.; Rothfuss, H.; Welle, A.; Richter, B.; Fischer, J.; Wegener, M.; Barner-Kowollik, C. Adv. Funct. Mater. 2014, 24,3571

(34) Jiang, Y.; Chen, J.; Deng, C.; Suuronen, E. J.; Zhong, Z. Biomaterials 2014, 35, 4969.

(35) Yu, Z.; Lin, Q. J. Am. Chem. Soc. 2014, 136, 4153.

(36) Kurra, Y.; Odoi, K. A.; Lee, Y.-J.; Yang, Y.; Lu, T.; Wheeler, S. E.; Torres-Kolbus, J.; Deiters, A.; Liu, W. R. Bioconjugate Chem. 2014, 25, 1730.

(37) Li, F.; Zhang, H.; Sun, Y.; Pan, Y.; Zhou, J.; Wang, J. Angew. Chem. Int. Ed. 2013, 52, 9700.

(38) Wang, J.; Zhang, W.; Song, W.; Wang, Y.; Yu, Z.; Li, J.; Wu, M.; Wang, L.; Zang, J.; Lin, Q. J. Am. Chem. Soc. 2010, 132, 14812.

(39) Li, J.; Huang, L.; Xiao, X.; Chen, Y.; Wang, X.; Zhou, Z.; Zhang, C.; Zhang, Y. J. Am. Chem. Soc. 2016, 138, 15943.

(40) Feng, W.; Li, L.; Yang, C.; Welle, A.; Trapp, O.; Levkin, P. A. Angew. Chem. Int. Ed. 2015, 54, 8732.

(41) Heiler, C.; Offenloch, J. T.; Blasco, E.; Barner-Kowollik, C. ACS Macro Lett. 2017, 6, 56.

(42) Li, Z.; Qian, L.; Li, L.; Bernhammer, J. C.; Huynh, H. V.; Lee, J.-S.; Yao, S. Q. Angew. Chem. Int. Ed. 2016, 55, 3254.

(43) Yang, N.; Rivas, C. J. Am. Chem. Soc. 1961, 83, 2213.

(44) Findlay, D. M.; Tchir, M. F. Journal of the Chemical Society, Faraday Transactions 1: Physical Chemistry in Condensed Phases 1976, 72, 1096.

(45) Porter, G.; Tchir, M. F. J. Chem. Soc. A 1971, 3772.

(46) Tyson, D. S.; Ilhan, F.; Meador, M. A. B.; Smith, D. D.; Scheiman, D. A.; Meador, M. A. Macromolecules 2005, 38, 3638.

(47) Tang, Q.; Wu, Y.; Sun, P.; Chen, Y.; Zhang, K. Macromolecules 2014, 47, 3775.

(48) Chen, L.; Xu, M.; Hu, J.; Yan, Q. Macromolecules 2017.

(49) Delaittre, G.; Guimard, N. K.; Barner-Kowollik, C. Acc. Chem. Res. 2015, 48, 1296.

(50) Gegenhuber, T.; De Keer, L.; Goldmann, A. S.; Van Steenberge, P. H. M.; Mueller, J. O.; Reyniers, M.-F.; Menzel, J. P.; D'hooge, D. R.; Barner-Kowollik, C. Macromolecules 2017, 50,6451

(51) Frick, E.; Schweigert, C.; Noble, B. B.; Ernst, H. A.; Lauer, A.; Liang, Y.; Voll, D.; Coote, M. L.; Unterreiner, A.-N.; BarnerKowollik, C. Macromolecules 2016, 49, 80.

(52) Nunn, A.; Minns, R.; Spesyvtsev, R.; Bearpark, M.; Robb, M.; Fielding, H. PCCP 2010, 12, 15751.

(53) Migani, A.; Blancafort, L.; Robb, M. A.; DeBellis, A. D. J. Am. Chem. Soc. 2008, 130, 6932.

(54) Frick, E.; Ernst, H. A.; Voll, D.; Wolf, T. J. A.; Unterreiner, A.-N.; Barner-Kowollik, C. Polym. Chem. 2014, 5, 5053.

(55) Voll, D.; Junkers, T.; Barner-Kowollik, C. Macromolecules 2011, 44, 2542.
(56) Günzler, F.; Wong, E. H. H.; Koo, S. P. S.; Junkers, T.; Barner-Kowollik, C. Macromolecules 2009, 42, 1488.

(57) Buback, M.; Günzler, F.; Russell, G. T.; Vana, P. Macromolecules 2009, 42, 652.

(58) Wiese, S.; Reidegeld, K. A.; Meyer, H. E.; Warscheid, B. PROTEOMICS 2007, 7, 340.

(59) Blasco, E.; Sugawara, Y.; Lederhose, P.; Blinco, J. P.; Kelterer, A.-M.; Barner-Kowollik, C. ChemPhotoChem 2017, 1, 159.

(60) Clovis, J. S.; Eckell, A.; Huisgen, R.; Sustmann, R. Chem. Ber. 1967, 100, 60 .

(61) Ober, C. K.; Cheng, S. Z. D.; Hammond, P. T.; Muthukumar, M.; Reichmanis, E.; Wooley, K. L.; Lodge, T. P. Macromolecules 2009, 42, 465.

(62) Kaupp, M.; Hiltebrandt, K.; Trouillet, V.; Mueller, P.; Quick, A. S.; Wegener, M.; Barner-Kowollik, C. Chem. Commun. 2016, 52, 1975.

(63) Barner-Kowollik, C.; Bastemeyer, M.; Blasco, E.; Patrick, M.; Delaittre, G.; Richter, B.; Wegener, M. Angew. Chem. Int. Ed., n/a.

(64) Gupta, N.; Lin, B. F.; Campos, L. M.; Dimitriou, M. D.; Hikita, S. T.; Treat, N. D.; Tirrell, M. V.; Clegg, D. O.; Kramer, E. J.; Hawker, C. J. Nat Chem 2010, 2, 138. 


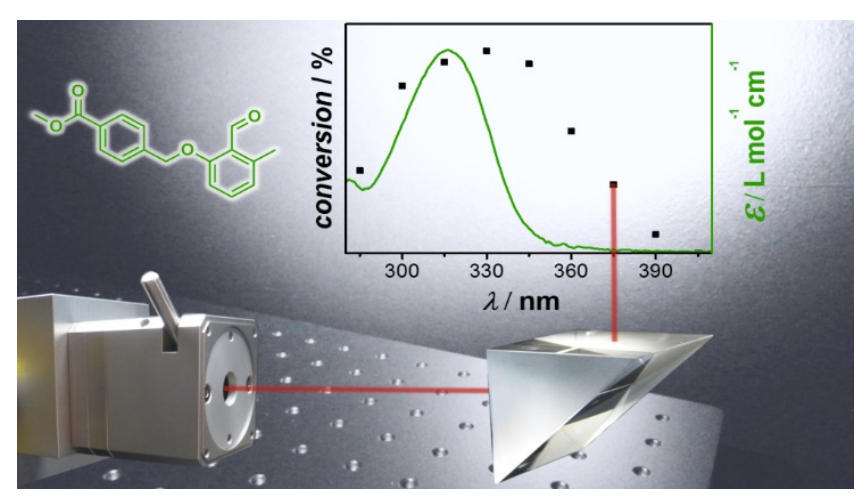

